\title{
A Study on Industry 4.0 Concept
}

\author{
Mr. Chaitanya Vijay Bidnur \\ Student of Padmabhoosan Vasantraodada Patil Institute of Technology, \\ Budhgaon (Sangli) (Maharashtra) \\ Branch: Mechanical (F.Y)
}

\begin{abstract}
Industry 4.0 is a strategic initiative recently introduced by the German government. The goal of the initiative is transformation of industrial manufacturing through digitalization and exploitation of potentials of new technologies. An Industry 4.0 production system is thus flexible and enables Individualized and customized products. The aim of this paper is to present and far-ciliate an understanding of Industry 4.0 concepts, its drivers, enablers, goals and limitations. Building blocks are described and smart factory concept is pre-scented. This paper presents a review on the advances of robotic and automation technology in achieving industry 4.0. Many companies, research centers, and universities acknowledge that robotics and automation technology is the basis of industrial manufacturing and an important driver for Industry 4.0. The current status of Industry 4.0 readiness of the German companies is presented and commented. Finally it is discussed if Industry 4.0 is really a disruptive concept or simply a natural incremental development of industrial production systems.
\end{abstract}

Keywords-Industry 4.0, Cyber-Physical Systems, Enterprise-Resource- Planning, Manufacturing Execution System.

\section{INTRODUCTION AND HISTORY:}

Industry 4.0 is a term often used to refer to the developmental process in the management of manufacturing and chain production. The term also refers to the fourth industrial revolution.

The term Industry 4.0 was first publicly introduced in 2011 as "Industry 4.0 " by a group of representatives from different fields (such as business, politics, and academia) under an initiative to enhance the German competitiveness in the manufacturing industry. The German federal government adopted the idea in its High-Tech Strategy for 2020. Subsequently, a Working Group was formed to further advice on the implementation of Industry 4.0.

In 2003, they developed and published their first set of recommendations. Their vision entailed that

"These Cyber-Physical Systems comprise smart machines, storage systems and production facilities capable of autonomously exchanging information, triggering actions and controlling each other independently. This facilitates fundamental improvements to the industrial processes involved in manufacturing, engineering, material usage and supply chain and life cycle management."

As the term "industry 4.0 " is not well -known outside the German -speaking area .It is worth looking at comparable ideas from a global perspective .Some commentator promotes a similar idea under the name of cyber physical systems ,smart factory, smart production, machine -to machine, advanced manufacturing, internet of everything or industrial internet. Industry 4.0 or fourth industrial revolution also refers to next phase in digitalization things looks to play a huge role that have the potential to feed informat

-ion into it and add value to manufacturing industry to realize a low-volume, high-mix production in a cost efficient way. It also involves the management and organization of the entire value chain process of the manufacturing industry. Various organization have been advocating industrial internet of things and industry 4.0 concepts to create smarter factories. Meanwhile according to the idea of industry 4.0 includes a wide variety of devices, from smart phones, gadgets, televisions and watches to household appliances which are becoming more flexible and intelligent.

\section{HISTORY:}

To be able to understand how Industry 4.0 became today's buzzword; a look at its predecessors might give us a perspective on how this revolution in particular is different. The following diagram shows a timeline of the evolution of manufacturing and the industrial sector in general (Source: Deloitte).

\section{THE FIRST INDUSTRIAL REVOLUTION}

The industrial revolution in Britain came in to introduce machines into production by the end of the $18^{\text {th }}$ century (1760-1840). This included going from manual production to the use of steam-powered engines and water as a source of power.

This helped agriculture greatly and the term "factory" became a little popular. One of the industries that benefited a lot from such changes is the textile industry, and was the first to adopt such methods. It also constituted a huge part of the British economy at the time

\section{THE SECOND INDUSTRIAL REVOLUTION}

The second one dates between 1870 and 1914 (although some of its characteristics date back to the 1850) and introduced pre-existing systems such as telegraphs and railroads into industries. Perhaps the defining characteristic of that period was the introduction of mass production as a primary means to production in general.

The electrification of factories contributed hugely to production rates. The mass production of steel helped 
introduce railways into the system, which consequently contributed to mass production. Innovations in chemistry, such as the invention of the synthetic dye, also mark such period as chemistry was in a rather primitive state then.

However, such revolutionary approaches to industry were put to an end with the start of World War I. Mass production, of course, was not put to an end, but only developments within the same context were made and none of which can be called industrial revolutions.

\section{THE THIRD INDUSTRIAL REVOLUTION}

Perhaps the third one is much more familiar to us than the rest as most people living today are familiar with industries leaning on digital technologies in production. However, the third industrial revolution is dated between 1950 and 1970 . It is often referred to as the Digital Revolution, and came about the change from analog and mechanical systems to digital ones.

Others call it the Information Age too. The third revolution was, and still is, a direct result of the huge development in computers and information and communication technology.

\section{IMPORTANCE \& SCOPE OF TECHNOLOGY IN} INDUSTRY 4.0:

Before diving into all the mentioned evolutions, integrations, cyber-physical evolutions, IoT elements, big data aspects and before looking at the origins of Industry 4.0, definitions, evolutions, global implications, similar initiatives, Industry 4.0 design principles, building blocks and the Reference Architecture Model Industry 4.0 a final word on technologies and people to wrap up this quick introduction.

It is important to note that Industry 4.0 is not just about those technologies. It also looks at the impact on and role of society and workers (e.g. collaboration between man and machine as with collaborative robots or cobots, new required skillsets of workers in industries amidst all these changes and, inevitably, the loss of jobs due to ongoing automation as mentioned - and how to tackle this major challenge).

Moreover, Industry 4.0 also has a strong focus on security. This does not just mean security of data and communication networks, data protection (including personal data protection, especially since the arrival of the General Data Protection Regulation, the ePrivacy Regulation and coming rules in several areas, including green facilities, energy and ecology, and again personal data in the wake of scandals that have emerged in consumer markets), cybersecurity in the broadest sense and industrial control systems security (ICS security) but also the protection and security of workers, industrial assets, critical infrastructure and 'physical security'.
As industrial assets and critical infrastructure (from critical power buildings to energy grids and more) get connected and attacks are on the rise in the traditionally rather isolated industrial environments the stakes and dangers of vulnerabilities and attacks are huge in industry 4.0 which requires an end-to-end 'security by design' approach.

This overall security challenge and certainly the ICS security and end-to-end cybersecurity challenge ranks high among the Industry 4.0 challenges and risks.

On an Industry 4.0 technology level, finally, on top of the mentioned technologies (or rather groups of technologies) such as IoT, big data, IT, OT, several security technologies (industrial cybersecurity, visual monitoring of critical facilities, wearables to monitor worker safety in hazardous environments), artificial intelligence $(A I)$, cloud, edge, fog and more you can add: additive manufacturing and 3D printing, digital twins, virtual and augmented reality (also in the context of digital twin simulation), advanced robotics and cobots, autonomous production, rapid application development, autonomous production, consistent engineering across the entire value chain, thorough data collection and provisioning, systems integration (cfr. vertical and horizontal integration), a vast range of networking and communication technologies, integration and connectivity standards/protocols, ways to make various IT and OT protocols interoperable and far more on the horizon, such as blockchain and technologies to not just meet demands for more bandwidth but also to power connected devices.

On the technological front it's important to look at the technologies underneath terms such as IoT but also to look at how it all connects, especially as on this technology level (where data and how you leverage, protect, exchange, enrich and even monetize it is at the center as you can see in the above list) the keyword is integration as well: from the integration of IT and OT to the integration of IoT and AI; big data, IoT and AI; blockchain and IoT.

New Industry 4.0 technologies, spanning mobile computing to cloud computing, have undergone vast development in the last decade and are now ready to be used as commercially available, interconnected systems within manufacturing - this is Industry 4.0. It holds the key to accessing real-time results and data that will catapult the industry into new levels of lean achievements.

The concept of Industry 4.0 however, is not a simple one. It envelops many technologies and is used in a variety of different contexts. There are five pieces that define Industry 4.0 at its core. Each piece is similar in nature but, when integrated together, create capability that has never before been possible. In an effort to understand Industry 4.0, the following five terms are explained as they contribute to the next industrial revolution: 


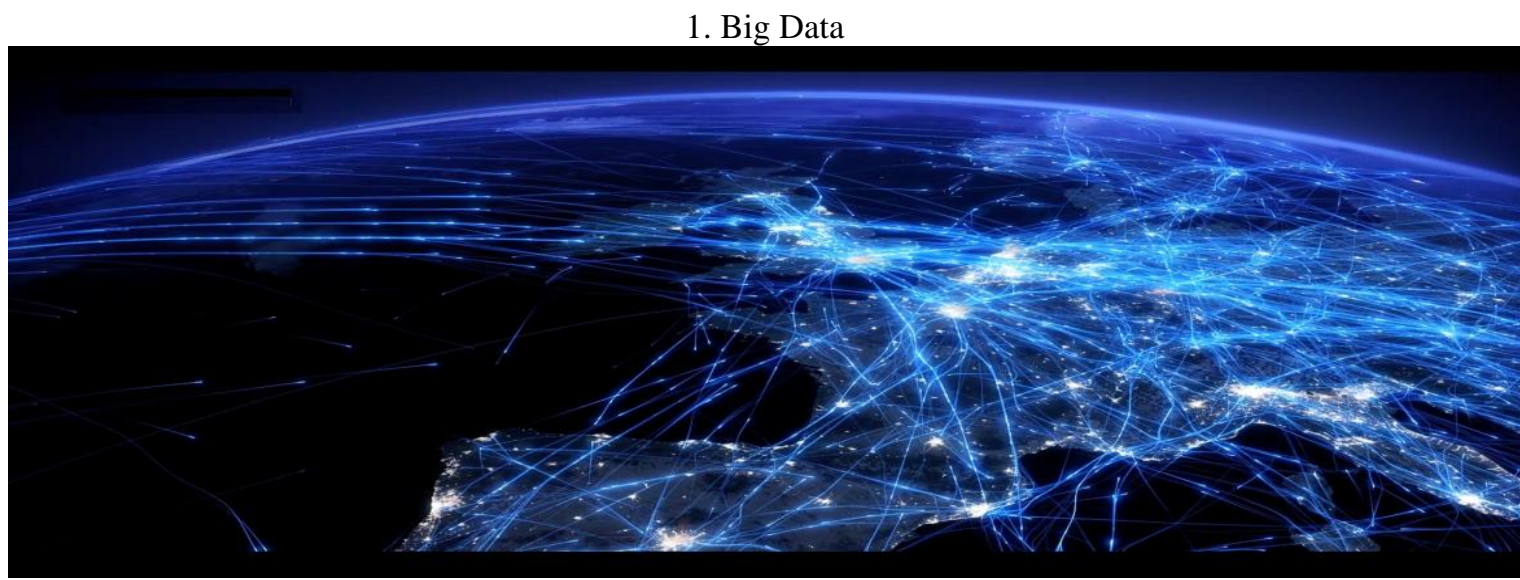

Photo credit: innovation-awards.nl

As per Forbes, Big Data is a collection of data from traditional and digital sources inside and outside your company that represents a source for ongoing discovery and analysis. Today data is collected everywhere, from systems and sensors to mobile devices. The challenge is that the industry is still in the process of developing methods to best interpret data. It's the evolution of Industry 4.0 that will change the way organizations and solutions within those organizations work together; teams will be able to make better, smarter decisions.

\section{SMART FACTORY:}

The concept of Smart Factory is the seamless connection of individual production steps, from planning stages to actuators in the field. In the near future, machinery and equipment will be able to improve processes through selfoptimization; systems will autonomously adapt to the traffic profile and network environment. Autonomous Mobile Robots (AMRs) are an integral part of the Smart Factory, as their autonomous intelligence connects the factory together, allowing seamless operations.

Leading by example is the Siemens Electronic Works facility in Amberg, Germany. Smart machines coordinate production and global distribution or a built-to-order process involving roughly 1.6 billion components. When the Smart Factory is achieved, it will represent a pivotal shift for Industry 4.0, as the revolution will begin to roll out across multiple verticals. Various markets spanning healthcare to consumer goods will adapt Industry 4.0 technologies initially modelled in the Smart Factory.

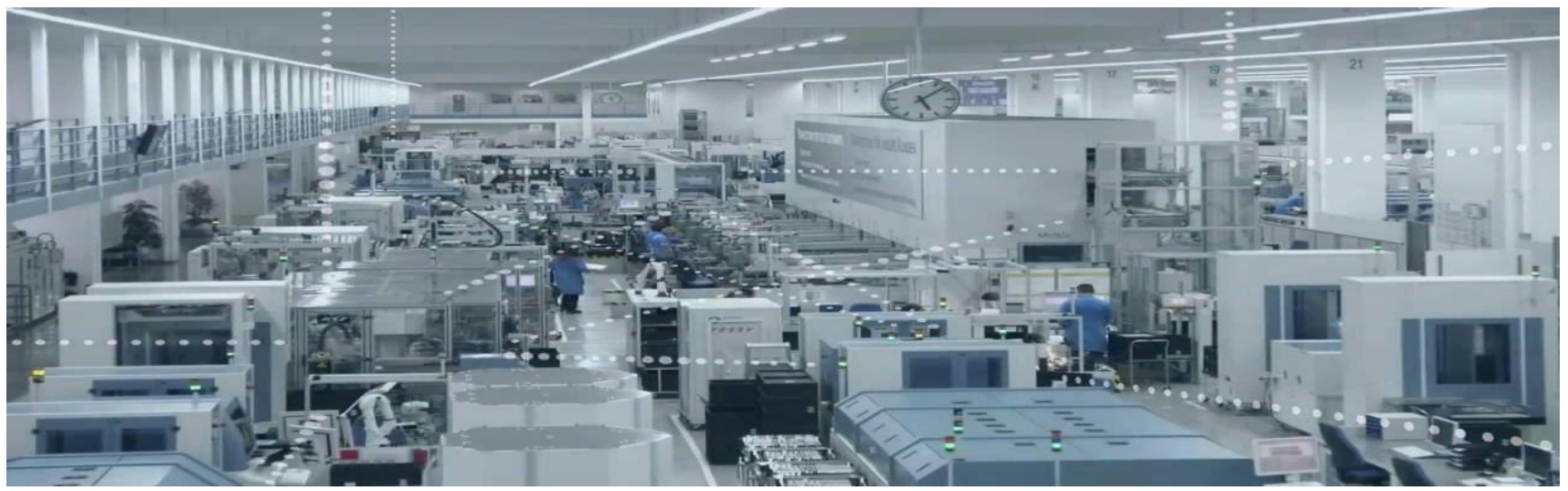

Photo credit: https://www.siemens.com/press/...

\section{CYBER PHYSICAL SYSTEMS}

Cyber physical systems are integrations of computation, networking and physical processes. Computers and networks monitor and control physical processes with feedback loops; the physical system reacts; the system uses software to interpret actions and tracks results. The notion centers on computers and software being embedded in devices where the first use is not computation; rather it is a loop of action and machine learning. 


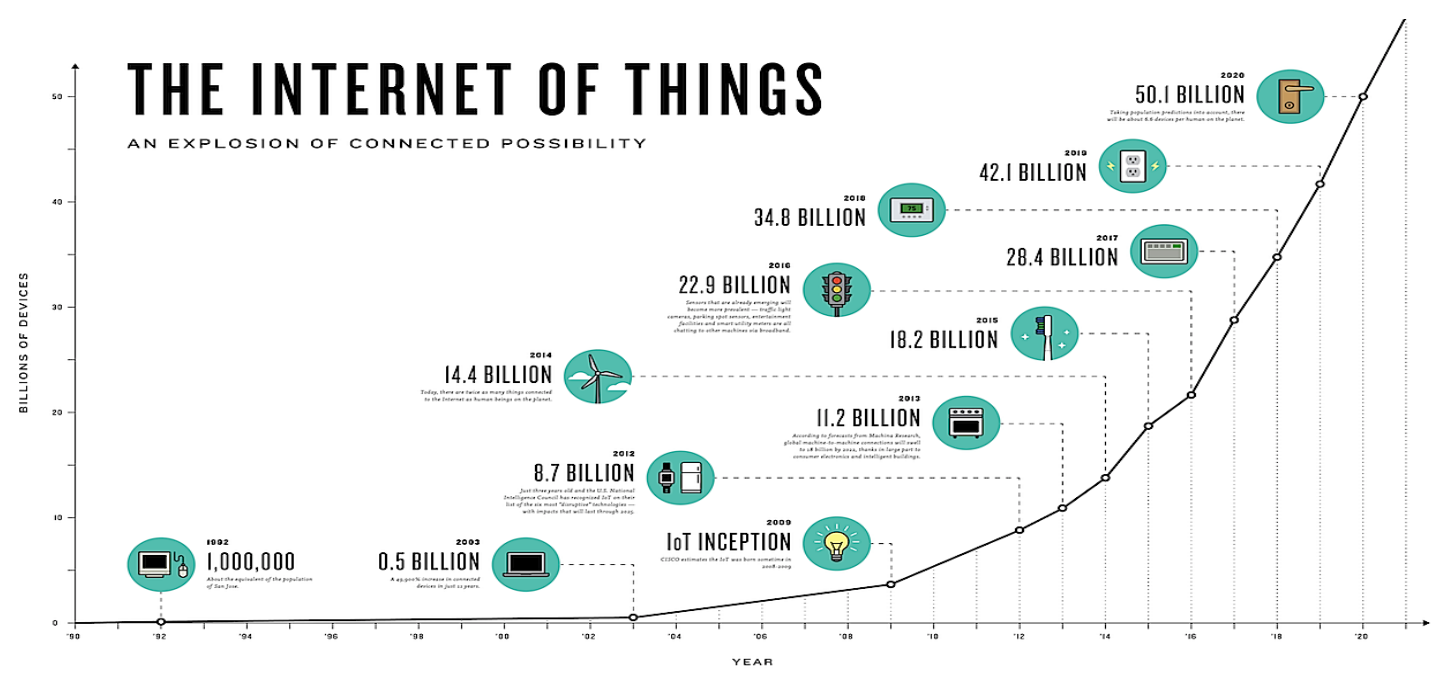

Photo credit: i-scoop.eu

The internet of things is a simple term for a grandiose concept. IoT is the connection of all devices to the internet and each other. As Wired said, "it's built on cloud computing and networks of data-gather sensors; it's mobile, virtual, and instantaneous connection." This interconnection will enable "smart factories" to take shape as equipment will use data to manufacture, move, and report and learn at astounding rates, efficiently.

\section{IMPORTANCE \& SCOPE OF TECHNOLOGY IN INDUSTRY 4.0:}

Before diving into all the mentioned evolutions, integrations, cyber-physical evolutions, IoT elements, big data aspects and before looking at the origins of Industry 4.0, definitions, evolutions, global implications, similar initiatives, Industry 4.0 design principles, building blocks and the Reference Architecture Model Industry 4.0 a final word on technologies and people to wrap up this quick introduction.

It is important to note that Industry 4.0 is not just about those technologies. It also looks at the impact on and role of society and workers (e.g. collaboration between man and machine as with collaborative robots or cobots, new required skillsets of workers in industries amidst all these changes and, inevitably, the loss of jobs due to ongoing automation as mentioned - and how to tackle this major challenge).

Moreover, Industry 4.0 also has a strong focus on security. This does not just mean security of data and communication networks, data protection (including personal data protection, especially since the arrival of the General Data Protection Regulation, the ePrivacy Regulation and coming rules in several areas, including green facilities, energy and ecology, and again personal data in the wake of scandals that have emerged in consumer markets), cybersecurity in the broadest sense and industrial control systems security (ICS security) but also the protection and security of workers, industrial assets, critical infrastructure and 'physical security'.

As industrial assets and critical infrastructure (from critical power buildings to energy grids and more) get connected and attacks are on the rise in the traditionally rather isolated industrial environments the stakes and dangers of vulnerabilities and attacks are huge in industry 4.0 which requires an end-to-end 'security by design' approach.

This overall security challenge and certainly the ICS security and end-to-end cybersecurity challenge ranks high among the Industry 4.0 challenges and risks.

On an Industry 4.0 technology level, finally, on top of the mentioned technologies (or rather groups of technologies) such as IoT, big data, IT, OT, several security technologies (industrial cybersecurity, visual monitoring of critical facilities, wearables to monitor worker safety in hazardous environments), artificial intelligence $(A I)$, cloud, edge, fog and more you can add: additive manufacturing and $3 \mathrm{D}$ printing, digital twins, virtual and augmented reality (also in the context of digital twin simulation), advanced robotics and cobots, autonomous production, rapid application development, autonomous production, consistent engineering across the entire value chain, thorough data collection and provisioning, systems integration (cfr. vertical and horizontal integration), a vast range of networking and communication technologies, integration and connectivity standards/protocols, ways to make various IT and OT protocols interoperable and far more on the horizon, such as blockchain and technologies to not just meet demands for more bandwidth but also to power connected devices. On the technological front it's important to look at the technologies underneath terms such as IoT but also to look at how it all connects, especially as on this technology level (where data and how you leverage, protect, exchange, enrich and even monetize it is at the center as you can see in the above list) the keyword is integration as well: from 
the integration of IT and OT to the integration of IoT and AI; big data, IoT and AI; blockchain and IoT.

\section{INDUSTRIAL ROBOTIC POTENTIAL IN INDIA:}

Sales of industrial robots in India reached a new record of 4,771 new units installed in 2018. That is an increase of $39 \%$ compared to the previous year (2017: 3,412 units). India now ranks eleventh worldwide in terms of annual installations. These are the findings of the report World Robotics 2019, presented by the International Federation of Robotics (IFR).

"India is one of the strongest growing economies among the emerging markets in Asia," says Junji Tsuda, President of the International Federation of Robotics. "Whilst the recent global results were quite moderate, India saw an impressive growth rate of $39 \%$ in 2018 . The number of robot installations has been growing rapidly for several years now. Between 2013 and 2018, India saw a compound annual growth rate of $20 \%$."

Prospects for further increasing robot installations are promising. The Indian GDP is expected to grow by more than 7\% in 2019 and companies across many industries intend to expand capacities. India has a young population, hence a strong workforce in need of jobs.

The country needs to expand its manufacturing industry to create more job opportunities. Higher wages and the rising share of affluent citizens in India are the main drivers of a growing and promising consumer market. The automotive industry remains the largest customer industry by far, with a share of $44 \%$ of total installations. But the strongest growth driver in 2018 was the general industry, increasing by $28 \%$, consisting of the rubber and plastics industry, the metal industry and the electrical/electronics industry. Robot use in non-automotive manufacturing is catching up with the automotive sector.

India now ranks eleventh in the global annual supply three places higher compared to the previous year and ahead of Singapore, Canada and Thailand. The operational stock of robots rose to about 23,000 units: $+21 \%$ compared to 2017. The country's automation potential is illustrated by a low robot density figure in the automotive industry: 99 industrial robots per 10,000 employees is less than a fourth of Indonesia's density (440 units) and far away from China (732 units) and the frontrunner Korea $(2,589)$.

\section{CURRENT STATE OF INDUSTRY 4.0 IN INDIA}

India has to move from manufacturing outfits of Industry 1.0 and 2.0 to Industry 4.0 and beyond. EEPC India in association of Department of Heavy industries (DHI) is raising awareness on the 4th Industrial Revolution to drive the Indian manufacturing to a 'Smart and intelligent Manufacturing' Hub said Mr. Ravi Sehgal at a Industry 4.0 session .

Smart Advanced Manufacturing and Rapid Transformation Hub (SAMARTH) - Udyog Bharat 4.0 is an Industry 4.0 initiative of Department of Heavy Industry, Government of India under its scheme on Enhancement of Competitiveness in Indian Capital Goods Sector. The initiative aims to raise awareness about Industry 4.0 among the Indian manufacturing industry through demonstration centres. Currently there are four centres which include Center for Industry 4.0 (C4i4) Lab Pune; IITD-AIA Foundation for Smart Manufacturing; I4.0 India at IISc Factory R \& D Platform; Smart Manufacturing Demo \& Development Cell at CMTI.

Indian Engineering Exports have been growing at a rate of $10 \%$ with variation, but the Engineering Exports as a percentage of ASEAN and World Exports is stagnating at $0.8-1 \%$ over the last $10-15$ years. This is because majority of engineering goods originated from low or middle level products. Department of Commerce, Ministry of Commerce and Industry has given to this apex engineering body a mandate to incarnate a Technology Centre to enable MSMEs to benefit from various new technologies. India also needs to close a quality gap faced with the best in class, and leapfrogging to newer technologies, will enable quality export products, hence EEPC India Technology Centre gains ground.

EEPC India Technology Centre in Bengaluru and also the one would be opened shortly in Kolkata, with a view to develop export product by providing a forum for Industry and Academia discussion and learning" Mr. Sehgal said at the seminar. It was a day long productive session where eminent speakers from DHI, IIT Kharagpur, ISI-Kolkata; CMERI- Durgapur; CMTI, C4i4 Pune motivated the stakeholders on the theme

In its pursuit to foster best-in-class manufacturing infrastructure in India, the "Make in India" initiative is spearheading wider adoption of 'Industry 4.0', the new buzzword for the combination of industry and the current Internet of Things (IoT) technology, in the country.

Banking on India's strength in Information Technology and a large workforce of IT professionals, the transformative journey of manufacturing through Industry 4.0 has already begun in the country. Under the Government of India's 'Smart Cities Mission', the projects to build 100 smart cities across India are being touted as the forerunners of the Industry 4.0 environment. ${ }^{1}$

Additionally, the Indian Institute of Science (IISc) is building India's first smart factory in Bengaluru with a seed funding from the Boeing Company. ${ }^{2}$ Bosch, a German auto component manufacturer will begin implementation of smart manufacturing at its 15 centres in India by $2018 .^{3}$ General Electric has invested USD 200 million in the facility in its only multi-modal factory in India where digitally interlinked supply chains, distribution networks, and servicing units form part of this intelligent ecosystem. With rapid development in the fields of information technology and hardware, the world is about to witness a fourth industrial revolution. ${ }^{4}$ the concept of 'Industry 4.0' is going to change the way India manufactures, designs and refurbishes the products. Driven by the power of big data, high computing capacity, artificial intelligence and analytics, Industry 4.0 aims to completely digitize the manufacturing sector. 


\section{CONCLUSION:}

In this paper, the background and development of the Industry 4.0 concept are presented. Although the concept is very comprehensive and complex, three main points can be identified:

- $\quad$ The Industry 4.0 concept is not limited just to the direct manufacturing in the company but it includes also a complete value chain from providers to customers and all enterprise's business functions and services

- The Industry 4.0 assumes broad support of an entire life cycle of systems, products and series, distributed both spatially and organizationally. The smart products are not smart only during the manufacturing process but they continue to provide the data about their state also during their lifetime. These data can be used for preventive maintenance; it can provide the manufacturer useful information about lifetime and reliability of their products.

- The Industry 4.0 is a specialization of the Internet of Things applied to the manufacturing/industrial environment. It assumes a real-time data collection leading to the issue of handling and analysing huge data and cybersecurity.

Industry 4.0 is new industrial revolution of the $21^{\text {st }}$ century, which enables companies to create "smarter" products and services by reducing costs and increasing efficiency, where the human factor is crucial for the application and the work is based on the existing literature in the area.

Smart Factory makes a solution which, due to the system's automated procedures, uncomplicated setup including simple, need-based installation and ultimately, high degree of scalability, can help companies in the manufacturing sector further optimize their processes and significantly boost their internal efficiency.

Five million workplaces could be lost due to digitalization in major industrialized nations. Also, it will quickly stop the market demand for the products made with outdated technologies due to lack of quality and such production would have to be shut down due to high costs and inefficiency.

Finally as Mr. Warren G. Bennis said:

"The factory of the future will have two employees; a human and a dog. The task of the human will be to feed the dog. The dog will have the task to dissuade the human to touch the automated systems."

\section{REFERENCES:}

\section{Research paper and articles:}

[1] INDUSTRY 4.0:A REVIEW ON INDUSTRIAL AUTOMATION AND ROBOTIC

[2] Author: Mohd Arman kamarul bahrin, Mohd Fauzi orthman, nor hayati azli, Muhamad farihan talib

[3] https://www.researchgate.net/profile/Fauzi_Othman/publication/3 04614356_Industry_40_A_review_on_industrial_automation_an d_robotic/links/57ac15aa08ae3765c3b7bab8.pdf

[4] SPECIAL FOCUS PAPER- INDUSTRY 4.0 CONCEPT:BACKGROUND AND OVERVIEW

[5] AUTHOR: ANDREJA ROJKO

[6] https://online-journals.org/index.php/ijim/article/viewFile/7072/4532

\section{WEBSITES:}

[1] https://www.researchgate.net/publication/303561107_Industry _40_the_Future_Concepts_and_New_Visions_of_Factory_of_ the_Future_Developmen

[2] https://economictimes.indiatimes.com/news/economy/policy/i ndustry-4-0-making-india-smart-and-intelligentmanufacturing-hub/articleshow/70585241.cms?from $=\mathrm{mdr}$

[3] https://en.wikipedia.org/wiki/Industrial_Revolution

[4] https://www.i-scoop.eu/industry-4-0/

[5] https://futureiot.tech/industrial-robots-market-in-india-grew39-in-2018/

[6] https://ottomotors.com/blog/5-industry-4-0-technologies

[7] https://en.wikipedia.org/wiki/Industry_4.0 